\title{
CARACTERÍSTICAS CLÍNICAS DE PACIENTES INTERNADOS EN EL HOSPITAL DE APOYO DE IQUITOS "CÉSAR GARAYAR GARCÍA" DURANTE LA EPIDEMIA DE DENGUE, ENERO-FEBRERO DE 2011
}

\author{
Víctor Fiestas Solórzano 1,a , Moisés Sihuincha Maldonado 2,a , Fernando Donaires Toscano 1,a , \\ Salomón Durand Velazco ${ }^{3, a}$, María García M. 1,b, Enrique Mamani ${ }^{4, c}$, \\ Juan Gómez de la Torre Pretell $5, d$
}

\begin{abstract}
RESUMEN
Se realizó una descripción clínica de pacientes con dengue confirmado internados en el Hospital de Apoyo de lquitos "César Garayar García” desde el 25 de enero al 05 de febrero de 2011. Según la actual clasificación de la OMS, los principales signos de alarma que motivaron hospitalización fueron: dolor abdominal (28/28) y vómitos persistentes (15/28); las causas de gravedad fueron: hipotensión (9/13), sangrado grave (6/13) y extravasación de plasma con dificultad respiratoria (4/13). La mediana de edad de los pacientes fue 22 años, sin embargo, 8/13 pacientes clasificados como dengue grave fueron menores de 16 años. En conclusión, se evidencia una mayor afectación de la población joven y gravedad en la presentación clínica a diferencia de epidemias anteriores, predominando el shock (hipotensión) por extravasación vascular, lo cual podría estar relacionado con la circulación de una variante emergente del DENV-2 con mayor virulencia.
\end{abstract}

Palabras clave: Dengue; Virus del Dengue; Dengue Grave; Perú (fuente: DeCS BIREME).

\section{CLINICAL CHARACTERISTICS OF PATIENTS ADMITTED TO HOSPITAL "CESAR GARAYAR GARCÍA", IQUITOS DURING THE DENGUE EPIDEMIC, JANUARY-FEBRUARY 2011}

\begin{abstract}
We conducted a clinical description of confirmed dengue cases admitted to the Hospital " César Garayar García" in lquitos from January 25 to February 5, 2011. According to current WHO classification, major warning signs leading to hospitalization were: abdominal pain $(28 / 28)$ and persistent vomiting (15/28), the causes of severity were: hypotension $(9 / 13)$, severe bleeding (6 / 13) and plasma extravasation with respiratory distress (4 / 13). The median patient age was 22 years, however, $8 / 13$ patients classified as severe dengue were under 16 years. In conclusion, unlike previous epidemics, we found a high involvement of young people and severity in the clinical presentation predominating a high frequency of shock (hypotension) and vascular leakage, which could be related to the circulation of an emerging variant DENV-2 which is more virulent.
\end{abstract}

Key words: Dengue; Dengue Virus; Severe Dengue; Peru (source: MeSH NLM).

\section{INTRODUCCIÓN}

En el Perú se ha identificado la circulación de los cuatro serotipos de dengue. En 1990 se introdujo el virus dengue serotipo 1 (DENV-1 $)^{(1-3)}$ a partir de la ciudad de lquitos, en 1995 se introdujo la cepa americana serotipo 2 (DENV-2) $)^{(3)}$, en el 2001 el virus dengue serotipo 3 (DENV-3) $)^{(4)}$ y en el 2008 el virus dengue serotipo 4 $(\mathrm{DENV}-4)^{(5)}$.

En diciembre de 2010 se inició en Loreto una epidemia de dengue con circulación de un nuevo linaje del DENV-2 genotipo americano/asiático, confirmado por el Instituto Nacional de Salud cuya transmisión se concentró en la ciudad de Iquitos (distritos San Juan, Belén, Iquitos y Punchana) que tiene una población de 400000 habitantes aproximadamente.

Frente a otras epidemias de dengue ocurridas en lquitos, en esta epidemia con circulación de un nuevo linaje del DENV-2 se evidenció un mayor porcentaje de hospitalización de pacientes con probable dengue, por lo cual consideramos necesario realizar un reporte de los principales hallazgos clínicos en estos pacientes.

\footnotetext{
Centro de Investigación de Enfermedades Tropicales "Maxime Kuczynski”- Instituto Nacional de Salud, Perú.

Hospital de Apoyo de Iquitos "César Garayar García" - Región Loreto, Perú.

Centro de Investigación de Enfermedades Tropicales de la Marina de los EEUU (NAMRU-6), Perú.

Laboratorio de Arbovirus - Instituto Nacional de Salud, Perú.

5 Universidad Nacional Mayor de San Marcos.

a Médico Infectólogo ${ }^{\mathrm{b}}$ Tecnólogo Médico ${ }^{\mathrm{C}}$ Biólogo a Médico Patólogo Clínico y Residente del III año en la Especialidad de Medicina de Enfermedades Infecciosas y Tropicales de la Universidad Nacional Mayor de San Marcos.
}

Recibido: 17-01-11 Aprobado: 09-03-11 


\section{EL ESTUDIO}

Desde el 23 de enero hasta el 05 de febrero de 2011 , médicos residentes y especialistas en enfermedades infecciosas y tropicales recolectaron en forma retrospectiva por anamnesis y prospectiva mediante una ficha clínica estandarizada, los signos y síntomas, así como los resultados de plaquetas y hematocrito de los pacientes, internados en el Hospital de Apoyo de lquitos "César Garayar García" con probable dengue con signos de alarma caracterizado por dolor abdminal intenso y continuo, vómitos persistentes, sangrado de mucosas, letargia o irritabilidad, hepatomegalia, signos de derrame pleural o ascitis o dengue grave definido por shock debido a extravasación de plasma (hipotensión) o sangrado grave según la última clasificación de la $\mathrm{OMS}^{(6)}$, y quienes tenían un tiempo de enfermedad menor o igual a cinco días.

Se realizó un análisis de las variables clínicas seleccionadas mediante el paquete estadístico SPSS versión 13.0, en aquellos pacientes que tuvieron diagnóstico de dengue confirmado por ELISA antígeno NS1, ELISAIgM o RT-PCR en tiempo real, realizado en el Centro de Investigaciones de Enfermedades Tropicales "Maxime Kuczynski" del Instituto Nacional de Salud; se excluyó aquellos pacientes que adicionalmente tuvieran otros diagnósticos como malaria o leptospirosis.

Asimismo, se analizaron las principales variables clínicas consignadas en la historia clínica de tres pacientes internadas en el Hospital de Apoyo de Iquitos
"César Garayar García" que fallecieron con diagnóstico confirmado de dengue. Durante el periodo de estudio no se produjo ningún fallecimiento.

\section{HALLAZGOS}

Se analizaron las variables clínicas de 41 pacientes hospitalizados con diagnóstico de dengue confirmado. De ellos, 28 (69\%) fueron clasificados como dengue con signos con signos de alarma y 13 (31\%) como dengue grave al alta de la hospitalización.

Los principales signos de alarma que motivaron hospitalización fueron: dolor abdominal (28/28) y vómitos persistentes (15/28). Las principales causas de gravedad fueron: hipotensión (9/13), sangrado grave (6/13) y extravasación de plasma con dificultad respiratoria (4/13).

La mediana del tiempo de enfermedad al momento de la hospitalización fue de tres días (rango: 0-5 días).

El promedio de edad fue de 28,4 años y la mediana de edad fue 22 años (rango: 7-57 años); sin embargo, destaca el hecho de que ocho de los trece pacientes clasificados como dengue grave fueran menores de 16 años. El 65,8\% fueron de sexo femenino.

Los signos y síntomas más frecuentes reportados en la anamnesis y hallados durante la evolución de los pacientes se muestran en la Figura 1, siendo los más comunes fiebre $(90,2 \%)$, cefalea $(80,5 \%)$, dolor muscular $(75,6 \%)$, dolor abdominal, nauseas $(70,7 \%)$,

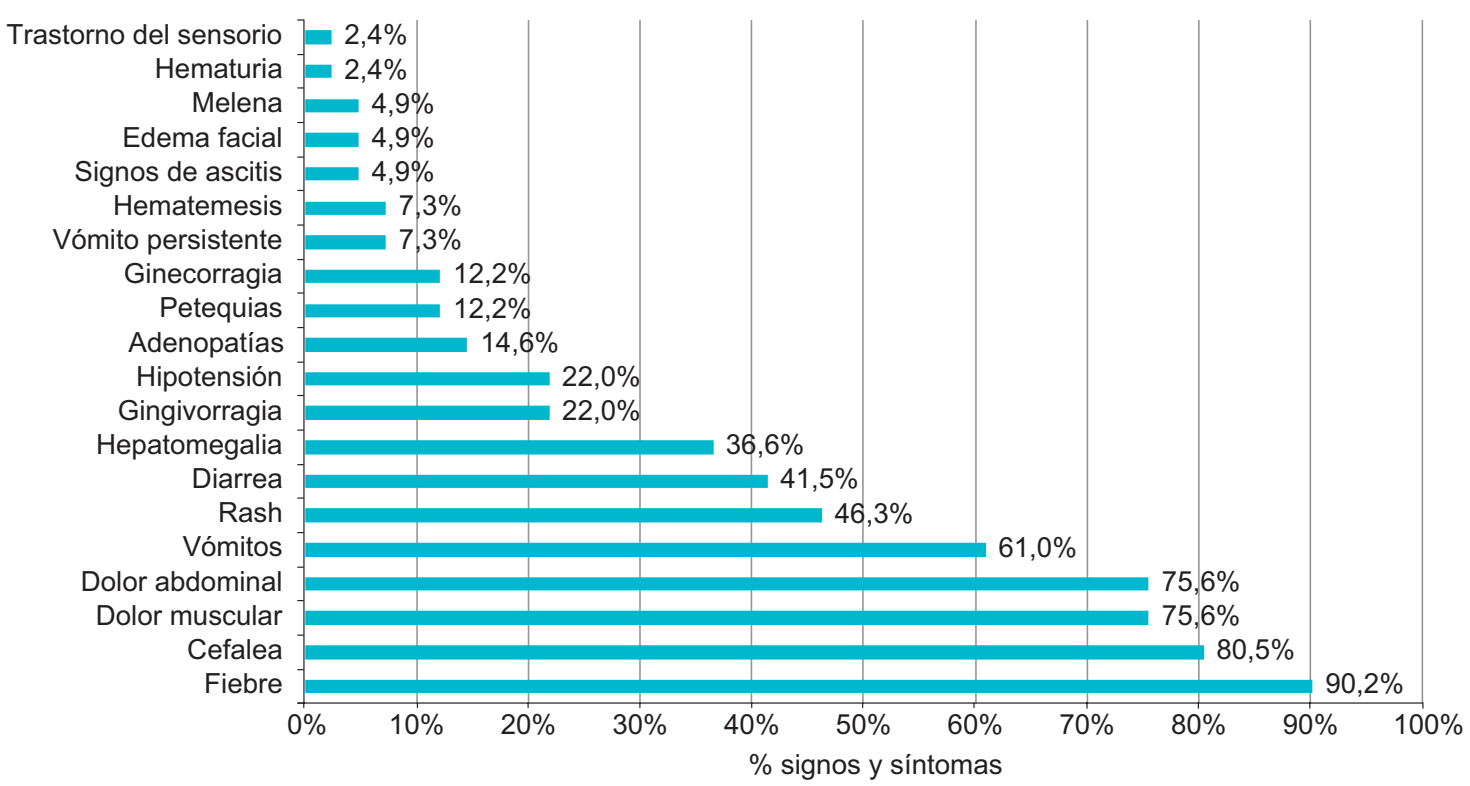

Figura 1. Principales signos y síntomas de pacientes hospitalizados con diagnóstico de dengue en el Hospital de Apoyo de lquitos “César Garayar García”, enero-febrero 2011. 


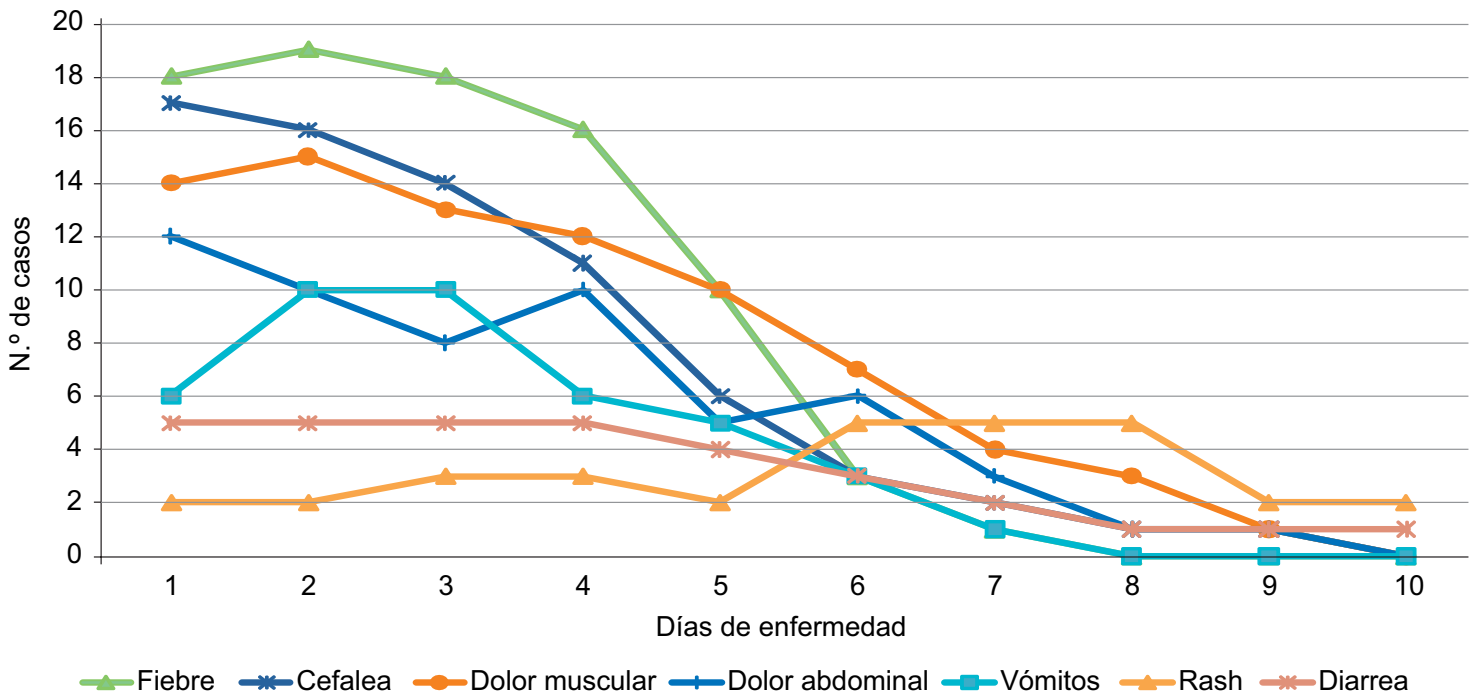

Figura 2. Evolución de los signos y síntomas por día de enfermedad en pacientes hospitalizados con diagnóstico de dengue en el Hospital de Apoyo de Iquitos "César Garayar García”, enero-febrero 2011.

prurito $(63,4 \%)$, vómitos $(61 \%)$, rash $(46,3 \%)$, lumbalgia $(43,9 \%)$ y diarrea $(41,5 \%)$.

La fiebre, cefalea y dolor muscular fueron los síntomas más frecuentes en los primeros tres días de enfermedad. En el $68,7 \%$ de los pacientes en quienes se presentó diarrea, esta se inició en los primeros dos días de enfermedad. El rash con prurito fue más frecuente a partir del sexto día de enfermedad (Figura 2).

En el examen clínico los hallazgos más frecuentes fueron: hepatomegalia (24\%), ascitis (12\%), esplenomegalia $(7 \%)$, adenopatías cervicales $(7 \%)$ y edema facial $(5 \%)$; la ascitis y el edema facial solo fueron registrados si se hallaban antes del inicio del tratamiento, ya que se consideró que estos signos podían encontrarse también por complicaciones del manejo (sobrehidratación).

En 30 pacientes $(73 \%)$ se determinó trombocitopenia (rango: 13 000-149 000), que se presentaba desde el segundo día de enfermedad, pero más frecuentemente desde el $4 .^{\circ}$ al $6 .^{\circ}$ día de enfermedad (figura 3 ). Todos los pacientes con sangrado grave cursaron con trombocitopenia, sin embargo solo el $40 \%$ de los pacientes que tuvieron trombocitopenia presentaron sangrado de mucosas.

En 33 pacientes $(80,5 \%)$ se determinó IgG por ELISA en los primeros cinco días, lo cual estaría relacionado con infecciones secundarias por virus dengue. Asimismo, mediante RT-PCR en tiempo real se evidenció DENV-2 en el $41 \%(17 / 41)$ y DENV-4 en el $5 \%(2 / 41)$ de los pacientes.
Con relación a los tres casos fatales con diagnóstico de dengue grave, confirmado mediante ELISA IgM, la Tabla 1 presenta los principales datos clínicos. Los tres casos tenían una condición de riesgo: gestante, antecedente de diabetes mellitus y cáncer de colon; el primero presentó sangrado grave postparto y los otros casos presentaron sepsis de origen urinario y pulmonar, respectivamente.

\section{DISCUSIÓN}

El dengue es una de las arbovirosis mas prevalentes en las regiones tropicales y subtropicales del mundo, que se ha incrementado notablemente en la región de las américas en los últimos años.

En el año 2009 se confirmaron más de 6000 casos de dengue clásico y 11 casos de dengue hemorrágico en el país ${ }^{(7)}$ según la clasificación anterior de la OMS, la cual ha demostrado tener alta especificidad pero baja sensibilidad para la identificación de los casos que requieren intervención ${ }^{(8)}$. En el 2010 con la utilización de la nueva clasificación de la OMS se confirmaron más de 11000 casos de dengue sin signos de alarma y desde la semana epidemiológica 44 se comenzaron a confirmar casos de dengue con signos de alarma y dengue grave en el país ${ }^{(9)}$.

En diciembre de 2010 se inició en Loreto una epidemia de dengue con circulación de un nuevo linaje del DENV-2 genotipo americano/asiático que hasta la semana epidemiológica siete confirmaron más de 1000 casos 


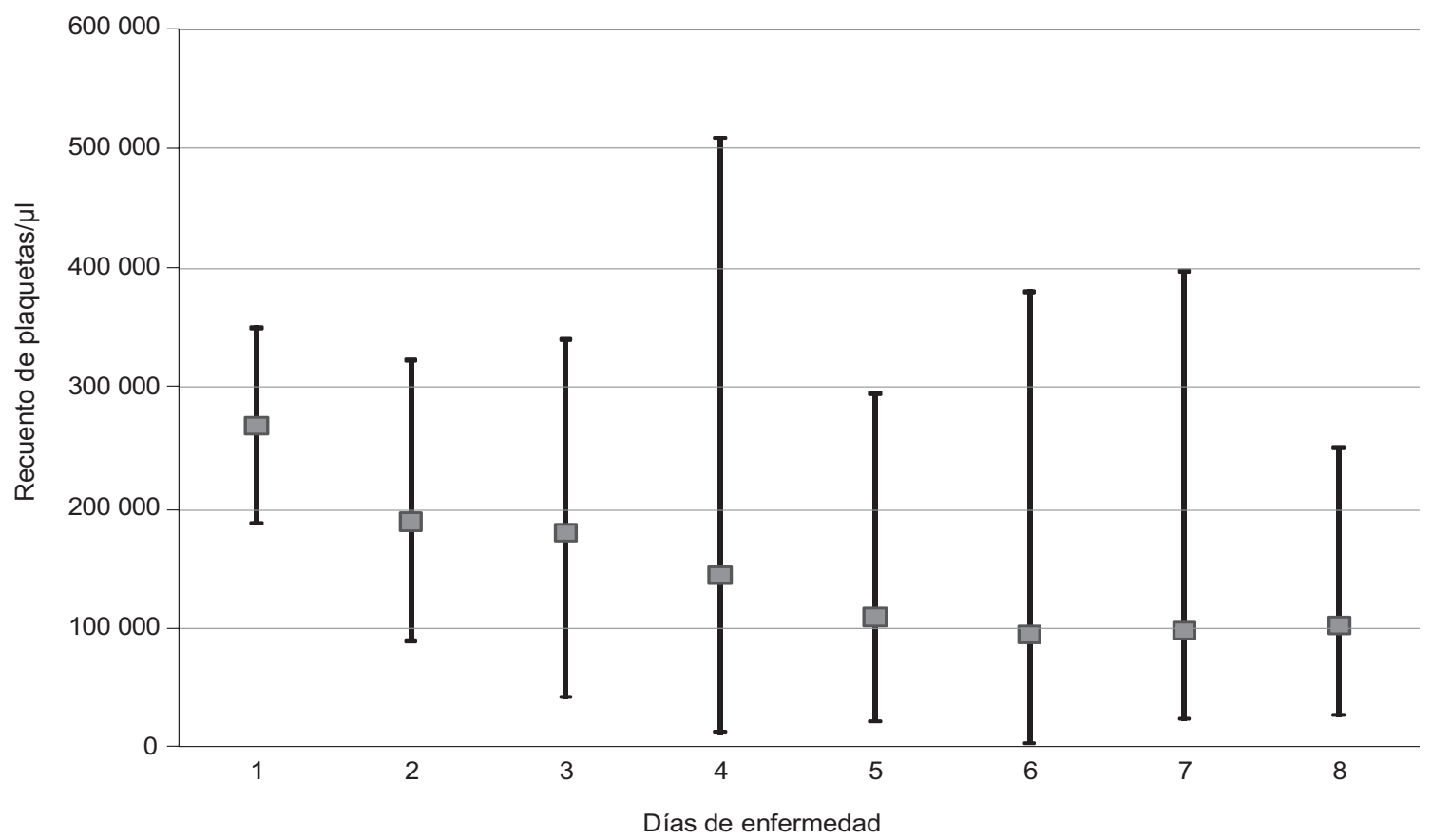

Figura 3. Evolución del recuento plaquetario en los primeros días de enfermedad de los pacientes hospitalizados con diagnóstico de dengue en el Hospital de Apoyo de Iquitos “César Garayar García”, enero-febrero 2011.

y 12 fallecidos ${ }^{(10)}$. Esta epidemia afectó principalmente a la población joven y se evidenció una mayor tasa de hospitalización y letalidad que en las epidemias ocurridas en la ciudad de lquitos en los últimos años.

En este estudio se evidencia una mayor severidad de los hallazgos clínicos en los pacientes hospitalizados que en epidemias anteriores, predominando el shock (hipotensión) por extravasación vascular, lo cual podría estar relacionado a la circulación de una variante emergente del DENV-2 con mayor virulencia; sin embargo, serán necesario estudios adicionales que permitan corroborar esta afirmación.

Los mecanismos por los cuales el virus dengue causa enfermedad severa aún no han sido aclarados completamente, debido a que es una enfermedad que solo se desarrolla en humanos y no contamos con modelos animales que permitan estudiar adecuadamente la virulencia de cada serotipo y genotipo del virus. Sabe- mos que factores virales e inmunológicos del huésped contribuyen en la patogenicidad, la inmunidad inducida por la infección provee protección específica contra un serotipo pero una infección secundaria por otro serotipo puede incrementar el riesgo de presentar dengue grave ${ }^{(11)}$.

Por otro lado, no hay evidencia de una rápida evolución y selección del virus dengue a diferencia de otros virus (VIH, influenza), por lo cual algunos investigadores opinan que la severidad clínica del dengue está en función de las características inmunológicas y genéticas de las poblaciones en el mundo(12).

Consideramos relevante este primer reporte de la evolución clínica de pacientes hospitalizados con dengue durante esta epidemia en lquitos, pues en lo sucesivo podrán verse afectadas otras regiones del país donde circule este virus emergente y el vector se encuentre presente.

Tabla 1. Características clínicas de pacientes fallecidos por dengue grave en Hospital de Apoyo de lquitos "César Garayar García”, enero 2011.

\begin{tabular}{|c|c|c|c|c|c|c|c|c|c|c|c|c|c|c|c|c|}
\hline \multirow[b]{2}{*}{ Caso } & \multirow[b]{2}{*}{ Edad } & \multicolumn{7}{|c|}{ Anamnesis } & \multirow[b]{2}{*}{$\begin{array}{l}\text { Tiempo de } \\
\text { enfermedad }\end{array}$} & \multicolumn{5}{|c|}{ Problemas durante hospitalización } & \multirow[b]{2}{*}{ Diagnósticos } & \multirow[b]{2}{*}{ Condición de riesgo } \\
\hline & & & Fiebre & Cefalea & Naúseas & Vómitos & Diarrea & Gingivorragia & & $\begin{array}{c}\text { Shock } \\
\text { descompesado }\end{array}$ & $\begin{array}{c}\text { Sangrado } \\
\text { grave }\end{array}$ & $\begin{array}{r}\text { Transtorno } \\
\text { del sensorio }\end{array}$ & Hiposlicemia & Trombocitopenia & & \\
\hline 1 & 82 & $\mathbf{F}$ & si & $5 i$ & si & No & $5 i$ & No & 7dias & Si & No & 5 & Si & No & $\begin{array}{l}\text { 1. De nue grave } \\
\text { 2. Ne umoniasdquitida en lacomunidas } \\
\text { 3. Falls multiorgánica }\end{array}$ & Cancer de colon \\
\hline 2 & 50 & $\mathbf{F}$ & Si & Si & si & Si & $5 i$ & No & 3dias & Si & No & 5i & si & No & $\begin{array}{l}\text { 1. Dengue grave } \\
\text { 2. Infección del tracto urinario } \\
\text { 3. Falls multiorgánica }\end{array}$ & $\begin{array}{l}\text { Diabetes mellitus } \\
\text { Hipertensión arterial }\end{array}$ \\
\hline 3 & 32 & $\mathbf{F}$ & Si & Si & No & No & No & Si & 6dias & Si & Si & si & No & Si & $\begin{array}{l}\text { 1. Dengue grave } \\
\text { 2. Post-cesareada por oligo-hidramnios } \\
\text { severo+distociafunicular } \\
\text { 3. Fally multiorgánica }\end{array}$ & Ninguno \\
\hline
\end{tabular}




\section{Fuentes de Financiamiento}

Instituto Nacional de Salud.

\section{Conflictos de Interés}

Los autores declaran no tener conflictos de interés en la publicación del presente artículo.

\section{REFERENCIAS BIBLIOGRÁFICAS}

1. CDC. Dengue epidemic--Peru, 1990. MMWR Morb Mortal Wkly Rep. 1991;8;40(9):145-7.

2. Phillips I, Need J, Escamilla J, Colan E, Sanchez S, Rodriguez M, et al. First documented outbreak of dengue in the Peruvian Amazon region. Bull Pan Am Health Organ. 1992;26(3):201-7.

3. Hayes CG, Phillips IA, Callahan JD, Griebenow WF, Hyams KC, Wu SJ, et al. The epidemiology of dengue virus infection among urban, jungle, and rural populations in the Amazon region of Peru. Am J Trop Med Hyg. 1996 Oct;55(4):459-63.

4. Kochel TJ, Aguilar P, Felices V, Comach G, Cruz C. Molecular epidemiology of dengue virus type 3 in Northern South America: 2000-2005. Infect Genet Evol. 2008;8:682-8.

5. Forshey BM, Morrison AC, Cruz C, Rocha C, Vilcarromero S, Guevara C, et al. Dengue virus serotype 4, northeastern Peru, 2008. Emerg Infect Dis. 2009 Nov;15(11):1815-8.
6. World Health Organization. Dengue. Guidelines for diagnosis, treatment, prevention and control. Geneva; WHO; 2009. Disponible en http://whqlibdoc.who.int/ publications/2009/9789241547871_eng.pdf

7. Ministerio de Salud del Perú. Situación del dengue en el Perú. Boletín Epidemiológico. 2009;18(52):984-6.

8. Srikiatkhachorn A, Gibbons R, Green S, Libraty D, Thomas S, Endy T, et al. Dengue Hemorrhagic Fever: The Sensitivity and Specificity of the World Health Organization Definition for Identification of Severe Cases of Dengue in Thailand, 1994-2005. CID. 2010;50:1135-43.

9. Ministerio de Salud del Perú. Situación del dengue en el Perú. Boletín Epidemiológico. 2010;19(52):1101-3.

10. Ministerio de Salud del Perú. Dengue. Boletín Epidemiológico. 2011;20(7).

11. Halstead SB. Dengue. Lancet. 2007;370:1644-52.

12. Rico-Hesse R. Dengue Virus Virulence and Transmission Determinants. Current Topics in Microbiology and Immunology. 2010;338:46-53.

Correspondencia: Victor Fiestas Solorzano.

Dirección: Instituto Nacional de Salud. Cápac Yupanqui 1400,

Lima 11, Perú.

Teléfono: (511)-617-6200.

Correo electrónico: vicfiso@yahoo.es

\section{Consulte la versión electrónica de la} Revista Peruana de Medicina Experimental y Salud Pública en

\section{wWW.scopus.com}

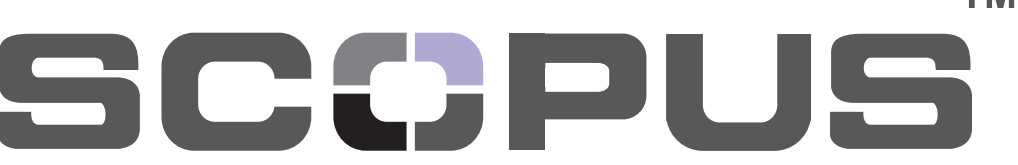

